\title{
Bright extreme-ultraviolet high-harmonic radiation from optimised pulse compression in short hollow waveguides
}

\author{
T. J. Butcher ${ }^{1}$ P. N. Anderson, ${ }^{1}$ R. T. Chapman,${ }^{2,3}$ P. Horak, ${ }^{1}$ J. G. Frey, ${ }^{2}$ and W. S. Brocklesby ${ }^{1}$ \\ ${ }^{1}$ Optoelectronics Research Centre, University of Southampton, SO17 1BJ, UK \\ ${ }^{2}$ Chemistry, Faculty of Natural and Environmental Sciences, University of Southampton, SO17 1BJ, UK \\ ${ }^{3}$ Central Laser Facility, STFC Rutherford Appleton Laboratory, Didcot, Oxfordshire, OX11 0QX, UK
}

(Dated: March 20, 2013)

\begin{abstract}
Multimodal nonlinear propagation dominates the evolution of intense laser pulses propagating in high pressure gas-filled capillaries used for high harmonic generation. A fully multimodal nonlinear propagation model is used to predict pulse evolution along such a capillary, and the length and pressure distribution optimised to produce the shortest pulses at the capillary output. This optimisation is shown theoretically to result in self compression of the pulse from $\sim 53 \mathrm{fs}$ to $\sim 7 \mathrm{fs}$, and shown experimentally to increase the flux of high harmonic radiation from the capillary by an order of magnitude over comparable capillary and gas jet designs.
\end{abstract}

PACS numbers: 42.65.Ky, 52.38.-r, 42.65.Re

At optical intensities above $>10^{13} \mathrm{~W} \mathrm{~cm}^{-2}$ the onset of tunnel ionisation allows for the observation of intense high-order harmonic generation (HHG) within atomic [1] and molecular gases [2]. Thanks to their small experimental footprint, high achievable photon energies and high coherence, lab-scale HHG-based sources have an array of potential applications if high photon flux in the extreme ultraviolet (XUV) and soft x-ray wavelength ranges can be generated.

High photon flux HHG requires many different criteria to be fulfilled. At relatively low intensities and gas densities, the fraction of the target gas which is ionised is low, and the plasma generated as a result of ionisation has a low density. Thus, propagation of the pump pulse is close to linear, and any effect of depletion of the ground state of the gas atoms can be neglected. Under these conditions, elegant solutions have been proposed for phase matching in gas jets using the Gouy shift[3] and in gas-filled capillaries using modal dispersion[4]. In capillaries, quasi-phasematching via linear propagation based techniques such as variation in capillary geometry[5] and mode-beating[6] have been demonstrated.

As laser intensities and gas pressures are increased, both the ionisation fraction and the plasma density can become large. In this case, ground state depletion and nonlinear propagation both act to make efficient generation harder to achieve. Generation in gas jets using very short $(<10 \mathrm{fs})$ pulses has been shown to improve harmonic yield at high intensities[7], by reducing ground state depletion at the peak of the pulse. The effect of nonlinear propagation on phase matching is also reduced, because the leading edge of the pulse sees much lower plasma density. Within a capillary waveguide, the generation of large densities of plasma causes significant nonlinear coupling between capillary modes, which redistributes energy between modes as a function of propagation distance. Thus phase matching based on the excitation of a single mode or combination of modes along the entire capillary will be severely degraded.

Nonlinear propagation effects can, however, be useful within the capillary waveguide. At low gas pressures ( $<10$ mbar) within the capillary, self compression of propagating pulses by a factor of $\sim 2.3$ has previously been observed[8] under conditions of high ionisation fraction but low plasma densities. Compression was assigned to spatiotemporal reshaping induced by ionisation.

In this letter we demonstrate the development of a high flux capillary-based HHG source in the high plasma density regime. The approach is based on theoretically rigorous modelling of ionisation-induced nonlinear mode coupling, which dominates the driver pulse propagation dynamics at high plasma densities. The model is used to optimise the gas pressure profile and length to produce pulses compressed from 53 fs to $\sim 7$ fs within the capillary. The experimentally-realised optimum capillary is shown to generate XUV with efficiencies an order of magnitude higher than previous capillary and gas jet-based sources around $29 \mathrm{~nm}$, using input pulses which are both longer and lower energy than previous experiments.

In order to predict the driver pulse propagation, a complex three-dimensional model based on a multimode-generalized nonlinear Schrödinger equation (MM-GNLSE) [9] was extended to include ionisation and plasma effects. The model predicts the full spatial, spectral, and temporal form of the pulse as it propagates along the capillary, taking into account factors including modal dispersion and losses (including higher-order dispersion), gas nonlinearity, plasma refractive index, self steepening, ionisation losses of the driver pulse, and the pressure profile along the capillary. The accuracy of this extended MM-GNLSE model in predicting the spatial and spectral evolution of an intense driver pulse within a high pressure gas-filled capillary has previously been experimentally verified[10].

The pressure profile within the capillary is determined by the introduction of the gas via holes in the capillary wall along its length. Figure $1(\mathrm{~d})$ shows a typical pressure profile for a capillary with two gas inlet holes, as calculated using computational fluid dynamics.

Maximisation of XUV flux requires that the pulse not 


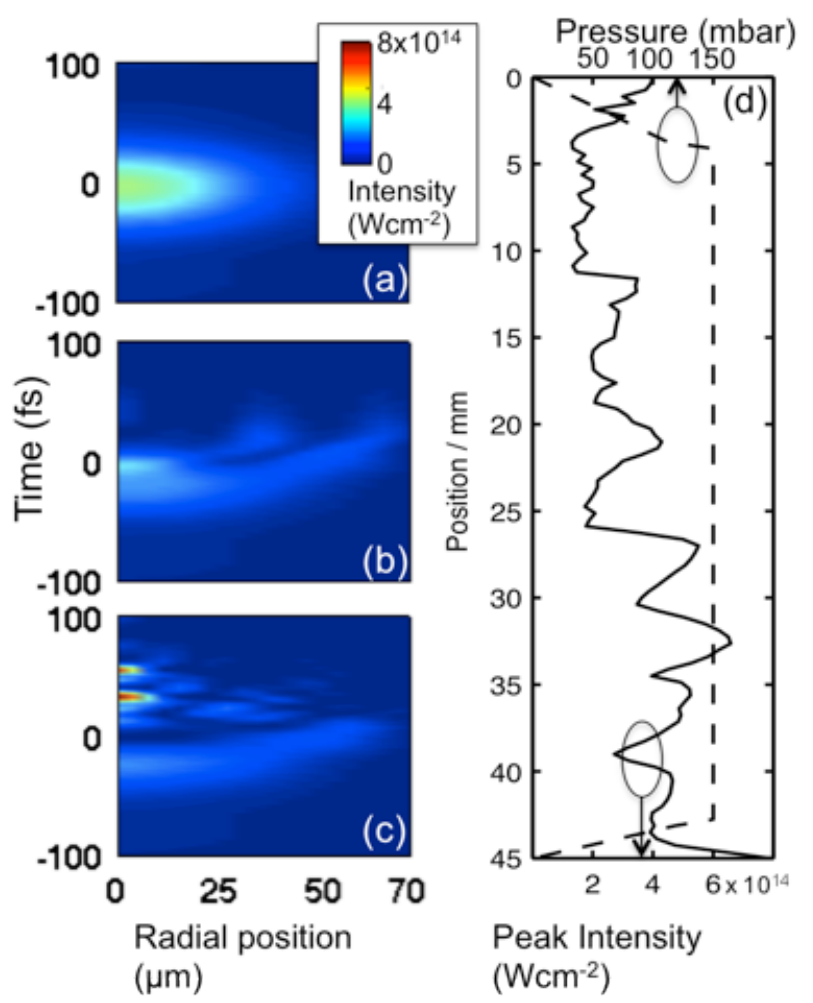

FIG. 1. (a) The predicted spatio-temporal profile of the $53 \mathrm{fs}$, $810 \mu \mathrm{J}$ Ti:sapphire driver pulse at the entrance of a $150 \mu \mathrm{m}$ core capillary filled with 150 mbar of Argon. (b) Modelled pulse at $\mathrm{z}=22.5 \mathrm{~mm}$ along capillary. (c) At $\mathrm{z}=45 \mathrm{~mm}$, nonlinear propagation effects produce $<7$ fs spatially and temporally compressed pulses. (d) Peak intensity of driver pulse as a function of position along the capillary (solid line). Dotted line shows schematic of pressure profile along capillary.

only has an ideal spatio-temporal intensity profile, but also that this intensity profile is reached at the ideal position along the capillary. In this work, the spatio-temporal compression is optimised at the far end of the capillary, thereby minimizing reabsorption of the XUV.

The extended MM-GNLSE model was used to simulate driver pulse propagation within a capillary with a variety of gas pressure profiles and capillary lengths. The input coupling parameters were determined from those measured experimentally, taking into account the laser phase front and beam parameters. As an example, Figure 1 shows the theoretical propagation along the capillary of an $810 \mu \mathrm{J}, 53 \mathrm{fs}$ input pulse centred at $790 \mathrm{~nm}$, with 150 mbar of argon present in the central region. The left three plots show the spatio-temporal distribution of the pulse at (a) $\mathrm{z}=0 \mathrm{~mm}$, the capillary entrance, (b) $\mathrm{z}=22 \mathrm{~mm}$, and (c) $\mathrm{z}=45 \mathrm{~mm}$, the capillary exit. The spatial distribution is shown on the $\mathrm{x}$-axis and the temporal distribution is shown on the y-axis. The right-hand image (d) shows the on-axis peak intensity of the pulse as a function of position along the capillary (solid line), together with a schematic representation of the pressure profile (dotted

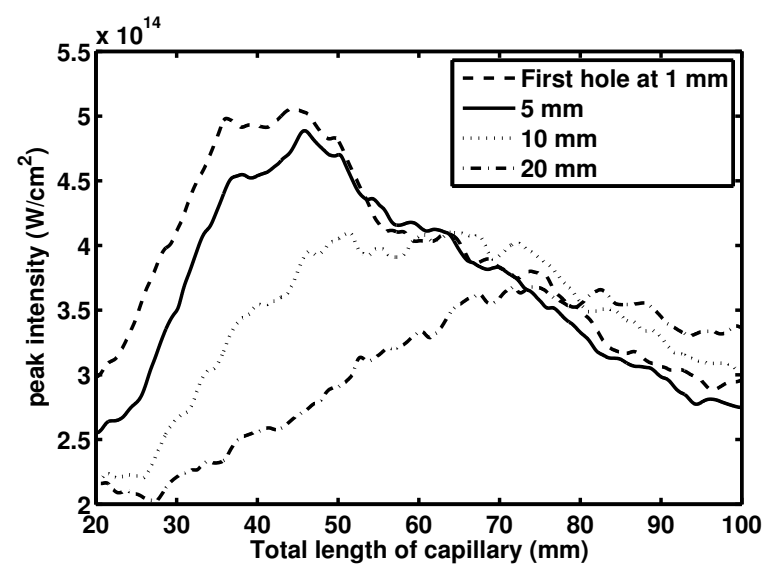

FIG. 2. Calculated pulse peak intensity, averaged through the last $20 \mathrm{~mm}$ of capillary, plotted vs capillary length for four different pressure profiles created by gas inlet holes at 1, 5, 10, and $20 \mathrm{~mm}$ from the capillary entrance. The peak pressure in these simulations was 150 mbar. A clear maximum is seen in the peak intensity for a capillary length of $45 \mathrm{~mm}$ and inlet hole positions at $1 \mathrm{~mm}$ and $5 \mathrm{~mm}$.

line). The high intensity peaks $\left(\sim 6-8 \times 10^{14} \mathrm{~W} \mathrm{~cm}^{-2}\right)$ observed in the final $20 \mathrm{~mm}$ are produced by the spatial and temporal compression of pulses, illustrated in figure $1(\mathrm{c})$. The temporal profile along the centre of the capillary in 1(c) shows two intensity spikes which are a factor of 10 higher than the rest of the pulse, and have FWHM of $\sim 7 \mathrm{fs}$. Pulse compression arises from the plasma generated by the leading edge of the driver pulse, which has a two-fold effect: the spatial change in refractive index due to the plasma scatters light into higher order modes, and the rapid change in refractive index with time leads to extensive spectral broadening. Once sufficient light has been scattered into higher order modes, the combined effect of nonlinear mode mixing, spectral broadening, and intermodal walk-off leads to the observed spatio-temporal compression of the driver pulse.

The model was used to optimise the spatiotemporal compression of the pulse at the capillary end, with the aim of improving HHG efficiency. Figure 2 shows the peak intensity of the spatiotemporally compressed pulse, averaged over the last $20 \mathrm{~mm}$ of the capillary, calculated for capillaries of different lengths for four different inlet hole positions at a particular maximum pressure (in this case, $150 \mathrm{mbar}$ ). The position of the front gas inlet hole is varied from $1 \mathrm{~mm}$ to $20 \mathrm{~mm}$ from the capillary entrance, keeping the second inlet hole very close to the back end of the capillary. The intensity averaged over the last $20 \mathrm{~mm}$ is the most relevant parameter because this is the region that contributes most to XUV generation around $29 \mathrm{~nm}$, because of reabsorption by argon gas[11]. A clear maximum can be seen at capillary lengths around $45 \mathrm{~mm}$, for holes placed close to the capillary entrance, illustrating the need to choose both length and pressure profile correctly for optimum performance.

In order to provide experimental confirmation of the 
predicted pulse compression and improvement of HHG flux, capillaries with internal diameter $150 \mu \mathrm{m}$ and varying lengths were used. The central region of the capillaries was filled with argon gas via a pair of lateral $100 \mu \mathrm{m}$ diameter gas inlet holes. Inlet holes were preferred over a segmented capillary design[4] because of their reduced scattering loss, and ease of fabrication and alignment. Two different capillaries were compared in experiments: a $7 \mathrm{~cm}$ capillary with gas inlets positioned $20 \mathrm{~mm}$ from the front and rear of the capillary, similar to that used in previous capillary generation experiments[2], and a 4.5 $\mathrm{cm}$ long capillary with inlets positioned 5 and $3 \mathrm{~mm}$ from front and rear respectively, which corresponds to the optimum hole positioning (although the optimum positioning of the front hole should be closer to the front of the capillary, $5 \mathrm{~mm}$ was the closest possible front hole position given fabrication limitations). Gas pressures up to 200 mbar could be introduced into the capillaries from a pressure-regulated supply. The capillaries were mounted within a vacuum chamber kept at $\sim 10^{-4}$ mbar. 53 fs laser pulses centred at $790 \mathrm{~nm}$, with a $1 \mathrm{kHz}$ repetition rate, were coupled into the capillary using a $0.5 \mathrm{~m}$ planoconvex lens. The ratio of beam spot size, $w$, to capillary radius, $a$, was 0.64 , giving optimum coupling into the lowest order, $E H_{11}$, mode[12]. Typical capillary throughput with no gas present was $\sim 70-90 \%$, indicating high coupling efficiency. The output of the capillary was filtered by two $200 \mathrm{~nm}$ free-standing $\mathrm{Al}$ film filters to separate the near infrared pulse from the XUV. An XUV CCD camera mounted on a translation stage could image the XUV beam, and high-order harmonic spectra generated by the capillary were measured at different gas pressures using a grazing incidence XUV spectrometer with range $20-60 \mathrm{~nm}$.

To provide an absolute value of the flux from the optimised capillary source from the measured values, rather than a comparative value, it is necessary to know the exact transmission of the optical elements between a source and detectors. The transmission of the background pressure of argon within the vacuum chamber was calculated to be $60 \%$.[11] The transmission through the aluminium filters was measured directly because variations in oxide layer thickness can affect filter transmission at $29 \mathrm{~nm}$ by a significant amount. The measured transmission through each single $200 \mathrm{~nm}$ aluminium filter was $13 \%$, and the overall transmission through the two filters and background argon gas was 1\%. Using the known XUV transmission and detector efficiency, the maximum flux generated by the optimised capillary, observed at $150 \mathrm{mbar}$, has a value of $1.52 \times 10^{9}$ photons/pulse, equivalent to an efficiency of $5 \times 10^{-6}$, and a brightness of $2.5 \times 10^{14}$ photons $\mathrm{s}^{-1} \mathrm{~mm}^{-2} \mathrm{mrad}^{-2}$. [13]. In comparison, the $7 \mathrm{~cm}$ capillary with non-optimum hole positioning produced a maximum flux of $3.9 \times 10^{7}$ photons/pulse, two orders of magnitude less than the optimised value, with the same input pulse and at the same gas pressure of 150 mbar. We note however, that large pressure dependent changes in flux were not observed with the $7 \mathrm{~cm}$ capillary.

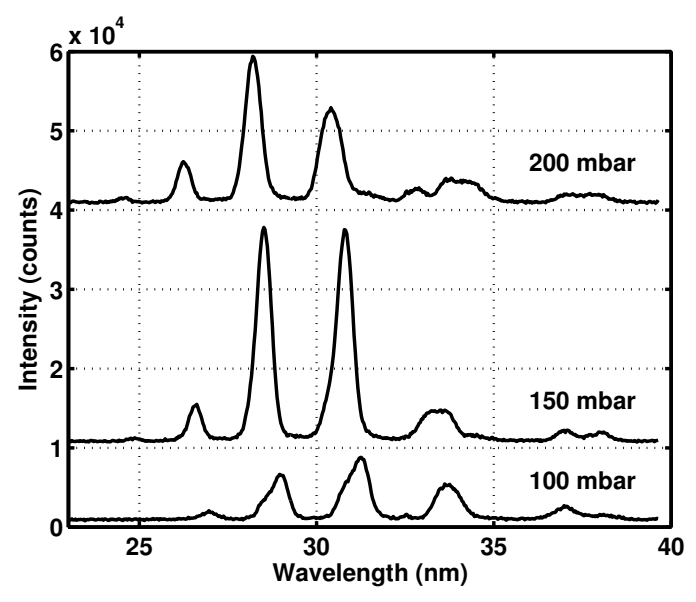

FIG. 3. The measured harmonic spectrum produced using the optimised capillary at pressures of 100, 150 and 200 mbar. The vertical scales are offset for clarity.

Comparison between flux values of different HHG sources is complex, because of the many experimental factors that can be varied, and many possible figures of merit. Practically, efficiency is important, as is absolute pulse energy needed for generation, as multi-mJ pulse lasers become increasingly expensive and typically have lower repetition rates, decreasing total flux/unit time. Comparisons must be made at similar generated wavelengths, as efficiency decreases as the generated photon energy increases.

Comparing this work to other capillary sources using similar pulse energies[14], we demonstrate here an increase in efficiency of a factor of $\sim 8$, and a factor of $\sim 6$ more photons per laser pulse, both achieved at shorter generation wavelengths $(29 \mathrm{~nm}$ vs $33-44 \mathrm{~nm})$ and with longer initial pulses ( $50 \mathrm{fs}$ vs $25 \mathrm{fs}$ ). Comparison to HHG sources based on loose focusing into gas jets at the same wavelength[15] shows that the generation efficiency at $29 \mathrm{~nm}$ demonstrated in this work is an order of magnitude higher, despite the use of multi-mJ pulses in reference [15]. Total flux levels per second from our source at $29 \mathrm{~nm}$ are also an order of magnitude higher, because of the increase in repetition rate made possible by the use of lower energy pulses. At slightly longer generation wavelengths $(\sim 35 \mathrm{~nm})$, efficiencies up to $3 \times 10^{-5}$ have been demonstrated using $2 \mathrm{~mJ}, 35$ fs pulses[16], and very high energy pulses $(16 \mathrm{~mJ})$ have been used to demonstrate efficiencies up to $6.4 \times 10^{-5}$ for the 15 th harmonic generated in $\mathrm{Xe}[17]$. In general, efficiency is increased at longer generation wavelengths and with higher pulse energies, although precise numerical factors for the increase of efficiency are difficult to calculate. The increase in efficiency produced by the use of optimised self-compression over directly comparable sources is clear.

Figure 3 shows the XUV spectra of the output of the optimised capillary for pressures of 100, 150, and 200 mbar. The XUV intensity is maximum at a pressure of 150 mbar. At this pressure two peaks, at $29 \mathrm{~nm}$ and 


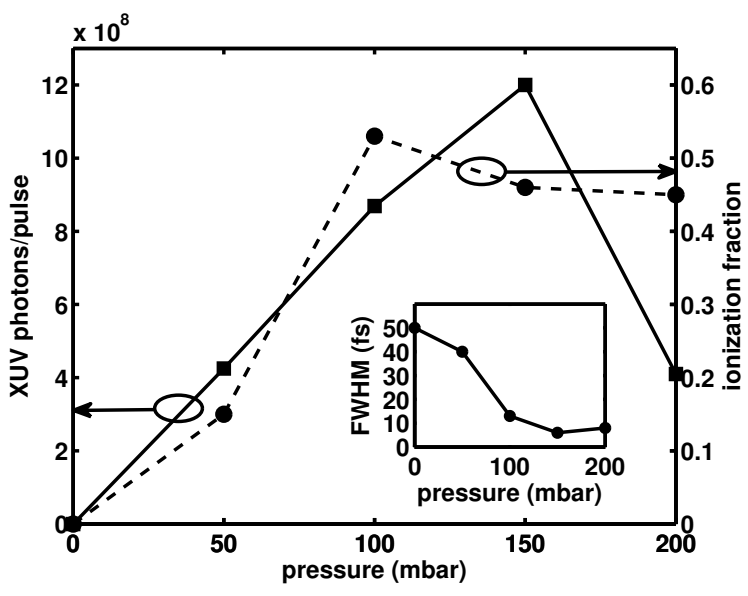

FIG. 4. Theoretical final ionisation level (circles) and measured total HHG flux (squares) vs. pressure. The inset shows the variation of theoretically-predicted pulse FWHM over the same pressure range.

$31 \mathrm{~nm}$, dominate the spectrum; these two peaks contain $75 \%$ of the total XUV signal, with a single harmonic peak containing $37.5 \%$ of the total flux. As a result the maximum flux generated by this capillary is $5.8 \times 10^{8}$ photons harmonic $^{-1}$ pulse $^{-1}$. The measured spectrum extends down to $\sim 25 \mathrm{~nm}$, at which point the Cooper minimum of argon suppresses the harmonic generation efficiency[18]. The compressed pulse near the end of the optimised capillary at 150 mbar is predicted to be 7 fs FWHM with a peak intensity of $\sim 8 \times 10^{14} \mathrm{~W} \mathrm{~cm}^{-2}$. Hence the measured harmonics are very far from the theoretical harmonic cutoff and retain their harmonic structure, and harmonics should be generated extending to energies higher than the Cooper minimum. No measurement in this spectral region was possible in these experiments. Experiments using neon gas, which does not show an equivalent minimum in this spectral region, measure harmonics down to $\sim 18 \mathrm{~nm}$, in accordance with the modelled intensities and the absorption edge of the Al filters.

The efficiency of HHG arises from the very short laser pulses generated at the end of the capillary. Figure 4 shows the variation with gas pressure of the measured HHG flux and the calculated ionisation fraction at the capillary end. At pressures where flux is highest $(\sim 150$ mbar), the ionisation fraction is much higher than would be predicted to be optimum for phase matching based on single mode propagation[4]; the inset shows that in this pressure region, the theoretically-predicted laser pulse length goes through a minimum, and is short enough that final ionisation levels do not dominate the phase matching. Moreover, ground state depletion on the pulse leading edge, where these harmonics will be generated, is reduced compared to longer pulses. We expect this effect to be even more significant for harmonics closer to the cutoff which are only generated at the pulse maximum. In the $7 \mathrm{~cm}$ capillary, less than $10 \%$ pulse compression is predicted over the entire pressure range by the model. The laser pulse length at the capillary end could not be measured accurately using the FROG systems available, as the high-order capillary modes which contribute to the very short pulse within the capillary are highly divergent outside the capillary. However, FROG measurements collecting only lower-order modes gave a pulse of length $15 \mathrm{fs}$, consistent with the modelled field distribution amongst the modes.

In summary, we have successfully used our MMGNLSE propagation model to design a short hollow capillary waveguide optimised for sub 10 fs pulse generation in the highly nonlinear propagation regime, and shown that the HHG flux from this capillary is significantly higher in both efficiency $(\times 8)$ and photons per pulse $(\times 6)$ than comparable HHG sources. A nonlinear propagation model was used to predict the necessary pressure profile and capillary length to temporally and spatially compress a $\sim 50$ fs input pulse to $<7$ fs within the capillary. The optimised capillary design was implemented experimentally, and produced a peak XUV flux of $\sim 1.5 \times 10^{9}$ photons/pulse around $29 \mathrm{~nm}$. We note that the capillary parameters here are suited to the particular gas and laser parameters of the experimental setup; however, the optimisation process is general throughout parameter spaces where nonlinear mode coupling dominates propagation.

The authors would like to acknowledge an EPSRC Basic Technology grant, and EPSRC studentships. The IRIDIS supercomputer facility at the University of Southampton was used for the modelling described in this paper.
[1] M. Ferray, A. L'Huillier, X. F. Li, L. A. Lompre, G. Mainfray, and C. Manus, Journal of Physics B: Atomic, Molecular and Optical Physics 21, L31 (1988).

[2] S. L. Stebbings, A. M. de Paula, E. T. F. Rogers, C. A. Froud, M. Praeger, B. Mills, D. C. Hanna, J. J. Baumberg, W. S. Brocklesby, and J. G. Frey, Journal of Physics B: Atomic, Molecular and Optical Physics 41, 145602 (2008).

[3] P. Salières, A. L'Huillier, and M. Lewenstein, Physical Review Letters 74, 3776 (1995).

[4] A. Rundquist, C. Durfee III, Z. Chang, C. Herne,
S. Backus, M. Murnane, and H. Kapteyn, Science 280, 1412 (1998).

[5] E. A. Gibson, A. Paul, N. Wagner, R. Tobey, D. Gaudiosi, S. Backus, I. P. Christov, A. Aquila, E. M. Gullikson, D. T. Attwood, M. M. Murnane, and H. C. Kapteyn, Science (New York, N.Y.) 302, 95 (2003).

[6] B. Dromey, M. Zepf, M. Landreman, and S. M. Hooker, Optics Express 15, 7894 (2007).

[7] T. Brabec and F. Krausz, Reviews of Modern Physics 72, 545 (2000).

[8] N. Wagner, E. A. Gibson, T. Popmintchev, I. P. Christov, 
M. M. Murnane, and H. C. Kapteyn, Physical Review Letters 93, 173902 (2004).

[9] F. Poletti and P. Horak, Journal of the Optical Society of America B 25, 1645 (2008).

[10] R. T. Chapman, T. J. Butcher, P. Horak, F. Poletti, J. G. Frey, and W. S. Brocklesby, Optics Express 18, 13279 (2010).

[11] B. Henke, E. Gullikson, and J. Davis, Atomic data and nuclear data tables 54, 181 (1993).

[12] R. Nubling and J. Harrington, Optical Engineering 37, 2454 (1998).

[13] This is a conservative estimate, due to a small amount of oil contamination present on the surface of the CCD, which may increase the actual values by $5 \%$.

[14] R. A. Bartels, A. Paul, H. Green, H. C. Kapteyn, M. M.
Murnane, S. Backus, I. P. Christov, Y. Liu, D. Attwood, and C. Jacobsen, Science (New York, N.Y.) 297, 376 (2002).

[15] J.-F. Hergott, M. Kovacev, H. Merdji, C. Hubert, Y. Mairesse, E. Jean, P. Breger, P. Agostini, B. Carré, and P. Salières, Physical Review A 66, 021801 (2002).

[16] S. Kazamias, D. Douillet, F. Weihe, C. Valentin, A. Rousse, S. Sebban, G. Grillon, F. Augé, D. Hulin, and P. Balcou, Physical Review Letters 90, 193901 (2003).

[17] E. Takahashi, Y. Nabekawa, and K. Midorikawa, Optics Letters 27, 1920 (2002).

[18] H. Wörner, H. Niikura, J. Bertrand, P. Corkum, and D. Villeneuve, Physical Review Letters 102, 103901 (2009). 